\title{
Huyendo de los extremos. Conciliación (Consilience) en la explica- ción del comportamiento violento humano
}

\author{
Avoiding the extremes. Consilience in the explana- \\ tion of human violent behaviour
}

\author{
JOSÉ SANMARTÍN ESPLUGUES \\ Universitat de València (España)
}

Recibido: 10-1-13

Aprobado definitivamente: 21-1-13

\section{RESUMEN}

En este artículo, a través del análisis de la conducta de sumisión característica de algunos tipos de víctimas de violencia, se muestra cómo, en el plano de la realidad hay interacciones ontológicas que, en el plano científico, deberían ser atendidas por interacciones epistémicas entre diversas teorías. Se sustenta de este modo una clase de consiliencia no basada en un micro-reduccionismo fuerte.

Proceder de este modo, dilucidar esas interacciones ontológicas en el marco de interacciones teórico-científicas, no sólo es lo coherente desde un punto de vista metodológico: es también lo más fructífero si se tienen en cuenta los grandes desarrollos que han experimentado ciencias en nuestro tiempo cuyos nombres ya delatan su carácter consiliente.

En este sentido, especial atención deberíamos prestar en el contexto del análisis de la conducta violenta a la neurociencia cognitiva como ámbito consiliente del saber que fomenta la colaboración de investigadores de áreas tan dispares como la neurofisiología, la ecología del comportamiento, la informática, la genética, la psicología y la psiquiatría.

\section{PALABRAS CLAVE}

CONSILIENCIA, REDUCCIONISMO SUBSTITUTIVO, REDUCCIONISMO COLABORATIVO, AMBIENTALISMO, BIOLOGISMO 


\begin{abstract}
In this paper, through the analysis of the submissive behavior characteristic of some types of violence, it shows how on the planes of reality there are ontological interactions which in scientific terms should be addressed by the epistemic interactions between various theories. It thus supports a class of consilience not based on a strong micro-reductionism.

Clarifying these interactions within ontological theoretical scientific interactions is not only consistent from a methodological point of view: it is also the most fruitful if one takes into account the major developments experienced by modern sciences whose names reveal their consilient nature.

We should therefore pay special attention to the context of the analysis of violent behavior and cognitive neuroscience as a consilient field of knowledge which fosters the collaboration of researchers from such diverse fields as neurophysiology, behavioral ecology, computer science, genetics, psychology and psychiatry.

KEYWORDS
CONSILIENCE, SUBSTITUTIVE REDUCTIONISM, COLLABORATIVE REDUCTIO-
NISM, ENVIRONMENTALISM, BIOLOGISM
\end{abstract}

\title{
I. INTRODUCCIÓN
}

En 1987, CUANDo PRESEnTÉ EN el CSIC en Madrid mi libro Los nuevos redentores (Barcelona, Anthropos), me mostraron su indignación unos cuantos eminentes biotecnólogos. ¡Hasta las ilustraciones del libro les molestaban! En una de las intervenciones, se me llegó a decir que era un redomado ambientalista, que ignoraba el verdadero papel que la biología jugaba en el comportamiento humano.

En 2004 y en el Recinto de Río Piedras de la Universidad de Puerto Rico, hablando en concreto de lo que ha sido mi objeto de investigación en estos últimos treinta años (la violencia) y sosteniendo posiciones parecidas a las que mantuve en Los nuevos redentores, la más relevante de las sociólogas de la isla llegó a insultarme diciendo que era un determinista biológico, precedida esta denominación de un grosero descalificativo.

Pero, ¿en qué quedamos? ¿Soy un determinista biológico o ambiental? La verdad es que no creo ser ni una cosa, ni la otra. Desde siempre he defendido algo que, incluso, me ha parecido del más absoluto sentido común. Nosotros somos animales que, por determinados rasgos biológicos, hemos desarrollado una notable cultura que, a su vez, ha generado un entorno al que estamos crecientemente adaptados. Hablar de este modo es típico de la posición que se ha denominado tradicionalmente «interaccionismo».

Lo cierto es que, actualmente, hay otras denominaciones que han hecho fortuna y que, desde una cierta ambigüedad, han embrollado este área. La más famosa, «consiliencia». 


\section{II. ¿QUÉ ES CONSILIENCIA?}

Ya saben que Consiliencia es el título de una de las últimas obras de Edward O. Wilson. Se trata de un libro monumental, por el tamaño y por el contenido. Es un canto al ideal de la ciencia unificada que, hundiendo sus raíces en la Grecia Clásica, encuentra su momento de máximo apogeo en el período de la Ilustración y revive con fuerza en la filosofía del Círculo de Viena.

Uno de los significados (quizá el dominante) del término «unificar»es juntar en una sola dos o más cosas que antes estaban separadas. La ciencia unificada es, entonces, el ideal de unir las ciencias en una sola. En los períodos y movimientos citados, esa sola ciencia ha sido la física. A veces, se ha preferido hablar, en general, de ciencias naturales, pero, en el fondo del discurso, siempre ha permanecido la idea de que la física es el gran espejo en el que los demás saberes han de mirarse y con el que tienen que (con)fundirse, si quieren ser aceptados en el mundo de la ciencia.

Pero «unificar» significa también juntar dos o más cosas en un grupo para alcanzar determinado fin, producir un cierto efecto, etc. No se juntan o funden dos o más cosas en una sola; se agrupan dos o más cosas, que no es lo mismo. Se puede unificar psicología y biología en biología, o se puede agrupar psicología y biología en psicobiología.

Lamentablemente, pese a las dimensiones del mencionado libro, Wilson no usa de manera unívoca el término «consiliencia». Desde luego, el padre de la Sociobiología asume como objetivo el ideal de la ciencia unificada, pero no clarifica si esa unificación significa sólo que podemos re-unir, es decir poner juntos conocimientos de distintas ciencias (naturales y sociales), o aunar por su raíz y fundir en su totalidad los conocimientos de tales ciencias. Según lo primero, la consiliencia sería meramente la vinculación de conocimientos de distintas disciplinas para crear un marco unificado de explicación. Según lo segundo, la consiliencia sería la fusión de las distintas disciplinas actuales para crear un solo marco de conocimiento. Como ya he dicho, creo que Wilson se debate entre una y otra posición. Una y otra, ciertamente, hacen uso del procedimiento llamado «reducción», pero su reduccionismo es de muy diferente cuño.

\section{REDUCCIONISMO}

Los partidarios de una sola ciencia practican un reduccionismo que, en terminología de Dennett, puede calificarse de codicioso. ${ }^{1}$ Sería mejor llamarlo «depredador». Es un reduccionismo que lo quiere todo. Lo abarca todo. Lo engulle todo. Nada queda fuera. Es un reduccionismo tragaldabas, basado en la idea de que tanto los entes como los métodos e hipótesis de una cierta disciplina

1 En inglés, «greedy reductionism». 
se identifican con y se explican o se substituyen respectivamente por los entes, métodos e hipótesis de otra disciplina que, por lo general, se encuentra en un nivel más básico (o inferior). Cuando lo que se reducen son cosas, esta posición recibe el nombre de «reduccionismo ontológico». Cuando lo que se reducen son métodos o hipótesis, se denomina «reduccionismo epistemológico». Un ejemplo paradigmático de este último tipo de reduccionismo lo constituyen quienes defienden que la biología no es más que física. El reduccionismo ontológico, fiel compañero de este reduccionismo epistemológico, consistiría en sustentar que los organismos no son otra cosa que conglomerados de substancias químicas y que éstas, a su vez, no son más que átomos. El organismo se disuelve en y es substituido por sus componentes últimos.

Por lo general, en este ámbito, se sustenta que lo reducido (sea ente, método o hipótesis) pertenece a un nivel de organización o complejidad más elevada que la propia del reductor. Y eso suele ser, repito, lo habitual cuando se habla de reduccionismo, término éste que, además, en boca de ciertos sectores de los científicos sociales, adquiere tonos muy peyorativos. Lo realmente curioso es que, en tales sectores, se practica con claridad total e, incluso, con orgullo y satisfacción un reduccionismo de signo contrario: no hacia abajo, hacia niveles más básicos de complejidad, sino hacia arriba. Se trata, en definitiva, de un reduccionismo en el que entes, métodos o hipótesis de un cierto nivel de complejidad tratan de identificarse o explicarse mediante entes, métodos e hipótesis de un nivel de complejidad superior. Un ejemplo paradigmático lo constituye el intento de reducir ontológicamente el individuo al colectivo, posición en cuyo nombre se llega a reducir epistemológicamente la antropología a la sociología. Otro ejemplo de este tipo de reducción a niveles superiores de complejidad lo ofrece el intento de explicar la conducta humana sólo y exclusivamente a partir de factores sociales.

Ciñéndome a este segundo ejemplo, no deja de ser curioso que reducir hacia arriba esté muy bien considerado (incluso, se tilda de progresista) en ciertos sectores del pensamiento y, en cambio, reducir hacia abajo tiene una pésima fama en esos mismos sectores. Eso es lo que lamentablemente suele suceder cuando ciencia e ideología se mezclan de manera insoluble.

Siguiendo con el hilo de mi argumentación, en lo sucesivo consideraré, por razones obvias, que el reduccionismo hacia la física es un micro-reduccionismo, mientras que el reduccionismo hacia la sociología es un macro-reduccionismo. En cualquiera de los dos casos, se trataría obviamente de un reduccionismo substitutivo.

Este reduccionismo substitutivo, por cierto, no ha logrado ni un solo éxito a lo largo de la historia de la humanidad. Nunca ha desaparecido una disciplina científica para ser reemplazada por otra de un nivel de complejidad inferior (o, como mucho, la desaparición ha sido meramente transitoria). 
Frente a este reduccionismo substitutivo cabe defender una variante no depredadora, una variante mucho más modesta, pero que ha llevado a desarrollos científicos muy notables, sobre todo en nuestro tiempo. Se trata del reduccionismo colaborativo que, desde un punto de vista epistemológico, no engulle unas disciplinas en otras, sino que atrae la atención científica hacia áreas en las que diversas disciplinas se interconectan. Son esas áreas en las que los límites entre tales disciplinas se tornan difusos e, incluso, irrelevantes. Lo importante es la interconexión misma, clave en múltiples ocasiones de progresos notables en el ámbito explicativo. Son áreas que, sobre todo en las últimas décadas, han ido constituyéndose en nuevas disciplinas de nombres híbridos, como genética molecular, biomedicina, neurociencia cognitiva o biología del comportamiento.

A este fenómeno de convergencia afortunada entre disciplinas científicas Wilson lo llama también «consiliencia» (Wilson, 1998): se trata de la vinculación de métodos e hipótesis a través de dominios científicos y a través de múltiples niveles anidados de complejidad.

\section{El REDUCCIONISMO COLABORATIVO COMO BASE DEL PROGRESO CIENTÍFICO}

A menudo, estas conexiones consilientes conducen a explicaciones que conllevan un cambio radical en la forma de enfrentarse a problemas tradicionales. Rand e Ilardi (2005) muestran, por ejemplo, cómo, en virtud de puentes consilientes, que ahora se han construido entre relevantes disciplinas médicas y biológicas, se ha logrado entender con gran detalle un trastorno como la fibrosis quística. Me detendré un momento en este punto.

La fibrosis quística es una de las enfermedades fatales más comunes. También ha sido denominada «mucoviscidosis» (del muccus, «moco», y viscosus, «pegajoso»), dado que se presenta como un síndrome de infección crónica respiratoria y cicatrización fibrosa, causado por tapones mucosos en los pulmones (primer nivel de complejidad).

Estos tapones mucosos tienen como causa el hecho de que el cuerpo enfermo produce mocos anormalmente viscosos (segundo nivel). ¿Por qué se producen estos tapones? Hasta hace muy poco tiempo, se desconocía su causa. Se sabía eso sí, que debían de tener alguna relación con el cloro y, en concreto, con el cloruro de sodio porque el sudor de los enfermos estaba muy salado. Una forma primitiva de diagnóstico consistía, obviamente, en tastar los mocos.

La aplicación de la química de la medicina permitió desarrollar un diagnóstico por identificación de electrolitos en el sudor: se administraba una substancia (pilocarpina) para estimular el sudor y se medía con un electrodo la concentración de sales en él. Esta técnica ha caído actualmente en desuso porque daba lugar a muchos falsos negativos. En cualquier caso, el desarrollo de la bioquímica ha permitido (tercer nivel de complejidad) encontrar una explicación para la enfermedad: la mucosa anormalmente viscosa, característica 
de la fibrosis quística, es causada por el transporte insuficiente de cloro a través de células epiteliales pulmonares, de ahí el sabor del sudor entre otras cosas.

Pero no acaban aquí las interconexiones consilientes afortunadas entre disciplinas. El desarrollo de la biología molecular (nueva consiliencia) ha permitido identificar una deficiencia en la proteína CFTR, deficiencia que es la causante del transporte inadecuado de cloruro a través de las células epiteliales pulmonares (cuarto nivel de complejidad).

Finalmente, el desarrollo de la genética ha posibilitado la identificación de las mutaciones que causan tal deficiencia en la proteína CFTR. De hecho, se han descrito más de 1.800 mutaciones. La mayoría de ellas son pequeñas deleciones, aunque con diferentes efectos, como cambios en el marco de lectura, cambios de aminoácidos, terminación prematura de la proteína...Estas mutaciones afectan a un gen (llamado por razones obvias «gen CFTR») que está ubicado en el brazo largo del cromosoma 7 y que ha sido secuenciado (quinto nivel de complejidad). El resultado es que la proteína CFTR está ausente o presente en proporciones muy bajas en los enfermos de fibrosis quística.

En el proceso explicativo descrito hemos ido micro-reduciendo, es decir yendo de arriba abajo a través de diversos niveles de complejidad, como se recoge en la imagen siguiente:

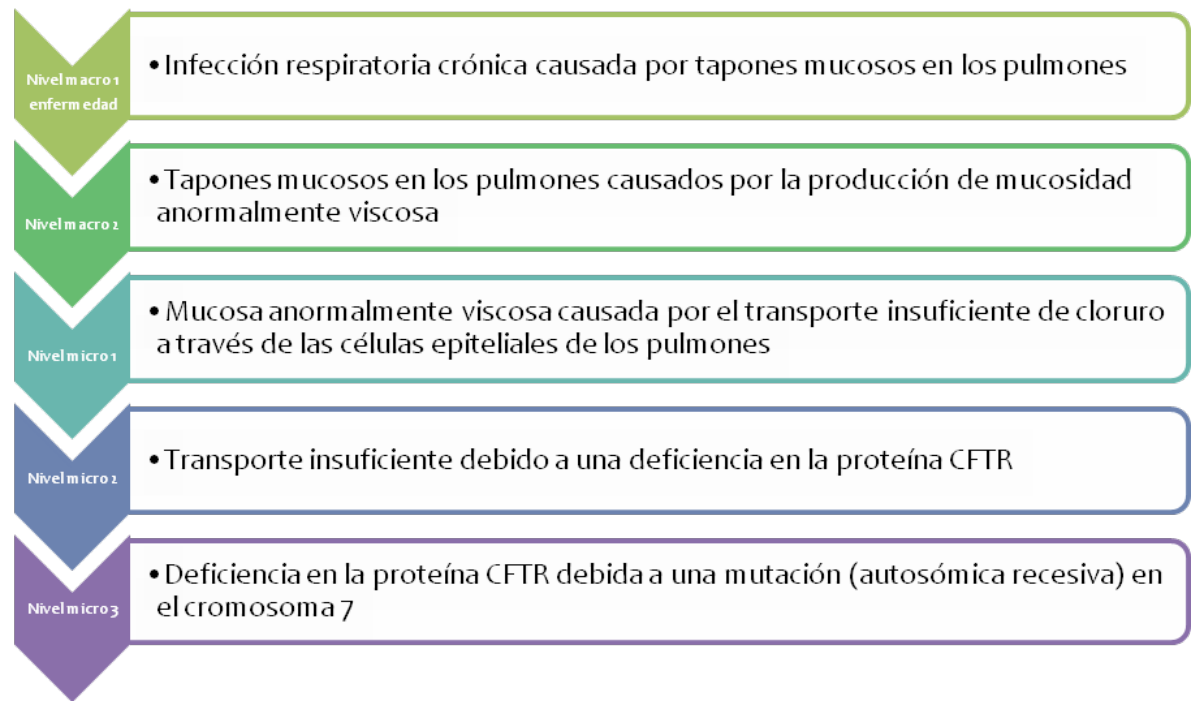

Como creo que ha quedado palmariamente mostrado, la existencia de este tipo de conexiones consilientes entre disciplinas a través de diferentes niveles 
de complejidad) posibilita un conocimiento científico (y, en este caso, una aplicación clínica) absolutamente inconcebible en su ausencia.

En casos como el descrito opera de modo totalmente claro el microreduccionismo colaborativo. Hechos de un nivel superior se dilucidan mediante descubrimientos y explicaciones en niveles de complejidad decreciente en los que operan disciplinas distintas. El resultado es la emergencia de un área de interconexiones que permiten avances notables desde un punto de vista teórico y, en ocasiones, práctico. En este sentido, se trata, como ya he dicho anteriormente, de interconexiones consilientes fructíferas que dejan fuera de sí partes (más o menos) amplias de las disciplinas afectadas. La medicina sigue siendo una disciplina, como la química o la biología. Pero una porción de ellas se interconecta generando disciplinas de nombres híbridos que se ocupan de realidades que se solapan, como «bioquímica», «psicobiología», etc. Para este tipo de consiliencia reservaré el calificativo de «colaborativa» 0 «prudente».

\section{LA CONSILIENCIA PRUDENTE Y EL COMPORTAMIENTO VIOLENTO}

La pertinencia de la consiliencia prudente es obvia si partimos de los hechos, aunque hablar de «hechos» no es algo que resulte epistemológicamente sencillo, ni siquiera para quienes, como yo, cultivamos un realismo crítico o constructivo.

Supongamos que vemos a una mujer que, ante agresiones periódicas de su compañero, se muestra sumisa e incapaz, por consiguiente, de adoptar cualquier medida que le permita alejarse de él. Desgraciadamente, se trata de un caso muy común, de las consecuencias de la violencia de género y que, en ocasiones, da lugar a posturas inadecuadas en la víctima. No hace mucho, en una cena con psiquiatras tuve que pararle los pies -científicamente hablando- a un par de ellos que estaban responsabilizando a tales mujeres de aguantar sin respuesta las vejaciones a que estaban sujetas. «iPor qué no rompen con el agresor?», me espetó el más famoso de los dos.

Creo que preguntas como la anterior, es decir cuestiones como ¿por qué se produce la violencia de género? ¿Por qué la víctima no rompe los lazos con el agresor? ¿Por qué se convierte en una víctima propicia?, sólo encuentran una respuesta rigurosa desde la consiliencia prudente.

En este sentido, pongamos el término «sumisión» en concreto en el centro (hablando físicamente) de una página hasta este momento en blanco.

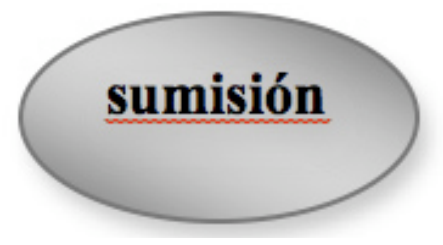


Podríamos decidir preguntarle a diversos tipos de científicos cómo explican que la sumisión en cuestión surja a menudo en el marco de la violencia de género.

Empezaré por los sociólogos. Las respuestas que suelen dar y que a continuación resumo son las que habitualmente yo mismo he recibido ante situaciones parecidas, en especial, durante estos últimos quince años. ${ }^{2}$

En el discurso sociológico, la violencia de género trata de explicarse a partir de la presencia de diversos factores ambientales que han incrementado la probabilidad de que tal conducta (la violencia) ocurra hasta convertirlo en seguro. Entre esos factores, el sociólogo suele distinguir:

a) Factores individuales, pertenecientes a la historia social del agresor en cuestión. ¿Cuál es su nivel de frustración? ¿Cómo ha interiorizado el rol social en el que ha sido educado y socializado?, etc.

Por lo general, el discurso sociológico dominante en este ámbito no suele plantearse cuestión alguna acerca del papel desempeñado en la conducta de la víctima por parte de factores individuales (a este respecto suele decirse que la víctima no tiene perfil).

b) Factores familiares, como el estilo educativo en el que el agresor ha sido criado por sus padres y parientes cercanos. Casi siempre se apunta hacia el estilo autoritario como responsable de este tipo de situaciones. El autoritarismo -se sostiene con frecuencia-, como estilo educativo caracterizado por un control excesivo y una gran falta de calor humano, se caracteriza porque incurre a menudo en prácticas violentas y el niño las aprende (aprendizaje social) porque las percibe como un medio expeditivo para conseguir lo que quiere.

En este punto, el sociólogo puede y suele aducir que las prácticas de crianza autoritarias producen niños violentos, pero niñas pasivas. Las niñas no aprenden socialmente a ser violentas, sino sumisas, porque esta conducta puede darles buen resultado: sufrirán menos.

2 Este tiempo abarca el período en que fui director del Centro Reina Sofía para el Estudio de la Violencia (1997-2010), período en que mis conocimientos teóricos y, en particular, prácticos en la problemática de la violencia de género se acrecentaron y me llevaron a actitudes y conductas que, al parecer, disgustaron a algunos sectores ideológicos. Se trata de los sectores que no dudaron en cerrar sin ningún tipo de escrúpulo intelectual un centro de referencia internacional como era el mencionado Centro Reina Sofía. 
c) Factores sociales, como las relaciones económicas, los esterotipos sociales (por ejemplo, de masculinidad o feminidad), las creencias patriarcales, las creencias en la eficacia del castigo, etc.

Estereotipos, prejuicios y creencias de ese tipo llevan a menudo a las víctimas a asumir como normal relaciones jerárquicas de sumisión y a justificar incluso el empleo de la violencia para el mantenimiento de las mismas.

En definitiva, es muy probable que el sociólogo trate de presentarnos la sumisión de la víctima como el resultado al que se llega al confluir un mal estilo educativo familiar y múltiples prejuicios y creencias distorsionadas acerca del papel social de la mujer.

Para reflejar este nivel de explicación sociológica trazaré un rectángulo en la parte izquierda de la palabra «violencia», así:

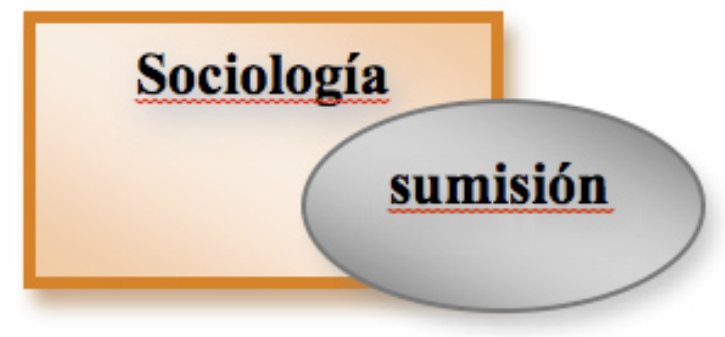

El discurso de la psiquiatría, solapándose en buena medida con el propio de la psicología (social y clínica, a la vez), es altamente probable que introduzca dos nuevos tópicos a la hora de explicar el hecho de que haya mujeres que no se rebelan ante su agresor, sino que tratan de adaptarse a sus pésimas condiciones de vida sometiéndose. Esos tópicos son el miedo y el síndrome de indefensión aprendida.

Como digo en un artículo que próximamente va a aparecer en Ludus Vitalis, el miedo inducido por la violencia aprisiona a la víctima hasta anularla, llegando en ocasiones a volverse insuperable. Y hay miedos insuperables, porque la única forma de vencerlos es con el raciocinio. Y la razón no opera en ese caso, porque el miedo lo impide. ${ }^{3}$ De una u otra forma la violencia pone bajo su dominio el miedo y lo optimiza.

3 Como he explicado en otros sitios con un ánimo consiliente [por ejemplo, Sanmartín Esplugues, J. 2002: La mente de los violentos. Barcelona: Ariel (3ª ed. 2011)], la amígdala, de la que dimanan las instrucciones bioquímicas que causan las reacciones fisiológicas integrantes 
Un miedo potenciado a un grado sumo es el que conduce a la denominada «indefensión aprendida», que sería mejor denominarla «indefensión condicionada». Este síndrome es inducido por la conducta metódica y sistemática de agresores que van frustrando todos y cada uno de los intentos de la víctima por salir de su situación, aterrorizándola hasta hacer del miedo ${ }^{4}$ una patología que lleva a la víctima, tras la toma de consciencia y consiguiente reflexión acerca de lo sucedido, a no oponerse a la agresión. La víctima ha interiorizado que no hay nada que hacer y que mejor es resignarse que enfrentarse. Estas ideas la conducen a una resignación forzada y al abandono de todo intento de escapar a la penosa situación por la que atraviesa.

Este tipo de indefensión condicionada se encuentra muy extendido entre toda clase de víctimas que se sienten desamparadas e incapaces de alcanzar sus metas vitales. Por ejemplo, las mujeres que sufren violencia de género, sobre todo aquellas cuyos maltratadores son cíclicos, ${ }^{5}$ suelen pasar por una fase (que, a veces, dura el resto de sus vidas) de resignación. Incluso, como suele ser común entre las víctimas condicionadas para la indefensión, justifican su situación recurriendo a todo tipo de falacias y creencias distorsionadas o no ajustadas a la realidad. «Algo habré hecho», «Me quiere tanto que...», «Son los celos, que son muy malos consejeros», «Él es bueno, pero no aguanta bien el alcohol», «Haga lo que haga, no lograré escapar», etc., son las sentencias en que se apoya la víctima para exonerar de responsabilidad al agresor, resignarse y racionalizar (falazmente) la victimización de que es objeto.

Para reflejar este nivel de explicación psiquiátrico-psicológica trazaré un rectángulo en la parte derecha de la palabra «sumisión», así:

de la emoción del miedo, si entra en fase de estrés, puede llegar a 'embargar' las acciones de la corteza prefrontal, en la que parecen residir las funciones conocidas como intencionalidad o toma de consciencia. Una amígdala hiperactiva, en definitiva, puede inducir una corteza prefrontal hipoactiva.

$4 \quad$ El miedo patológico se mezcla a menudo con la ansiedad patológica. El miedo se produce ante estímulos identificables (reales o imaginados). La ansiedad (patológica) es una sensación difusa de angustia o miedo y consiguiente deseo de huir ante circunstancias difíciles de vincular con fuentes tangibles de estimulación.

5 Entre los maltratadores de mujeres los hay, al menos, de tres tipos: psicopáticos, agresivo-pasivos y cíclicos (véase, por ejemplo, Sanmartín Esplugues, J. (2008), El enemigo en casa. Barcelona: Nabla). En el maltrato perpetrado por un agresor cíclico alternan fases de violencia con fases de reconciliación (incluso, de luna de miel). Esta alternancia suele generar en las víctimas un síndrome muy similar al de Estocolmo. 


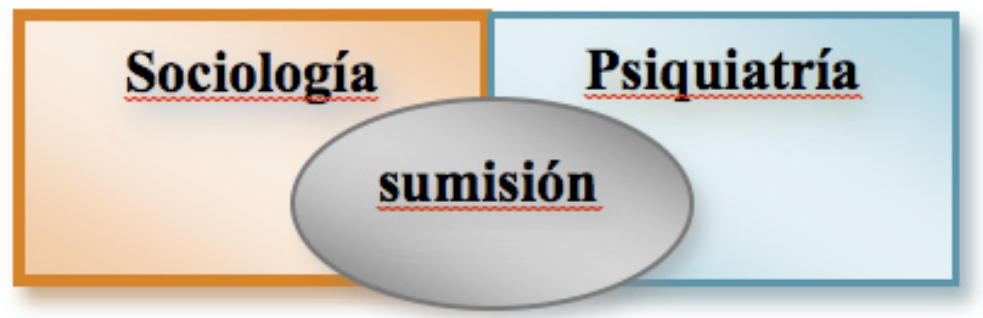

Le toca al turno ahora a los biólogos y, más en concreto, a los neurobiólogos.

Lo habitual en contextos como el presente es que se dediquen a diseccionar las diversas partes componentes de la conducta violenta en cuestión, del miedo y del síndrome de indefensión aprendida, y que traten de correlacionarlas con diversas estructuras del cerebro. Así, por ejemplo, supongamos que es un día lluvioso y que una mujer que está siendo víctima de violencia de género oye que se abre la puerta de su casa y la voz airada de su compañero maldiciendo por haber tropezado con el felpudo. Luego, ella verá una sombra en la puerta de la habitación en que se encuentra. ¿Qué interpretará un neurobiólogo que está sucediendo en el cerebro de los potenciales víctima y agresor? Más o menos lo siguiente:

1. El input visual (rostro del compañero) debe haber llegado, a través del nervio óptico de la víctima, a su tálamo que, a su vez, habrá remitido tal input a la amígdala (siguiendo una vía corta) y a la corteza occipital (siguiendo una vía larga).

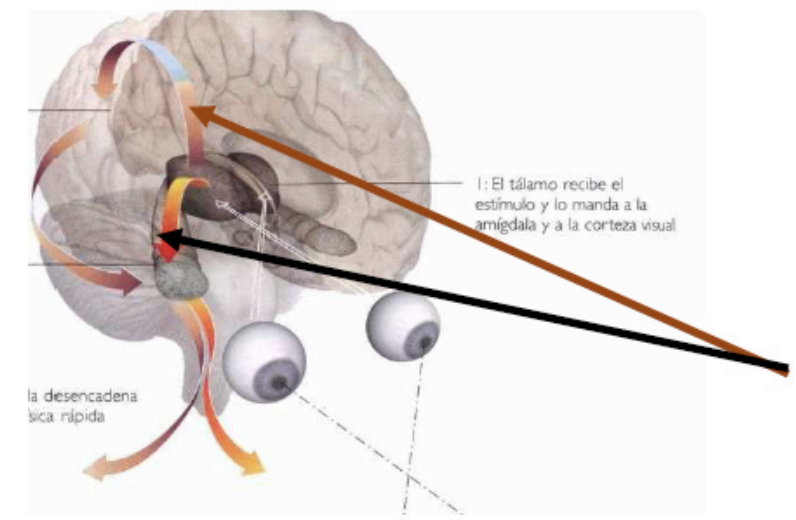


2. De la amígdala, tal input en bruto se dirigirá hacia estructuras del troncoencéfalo:

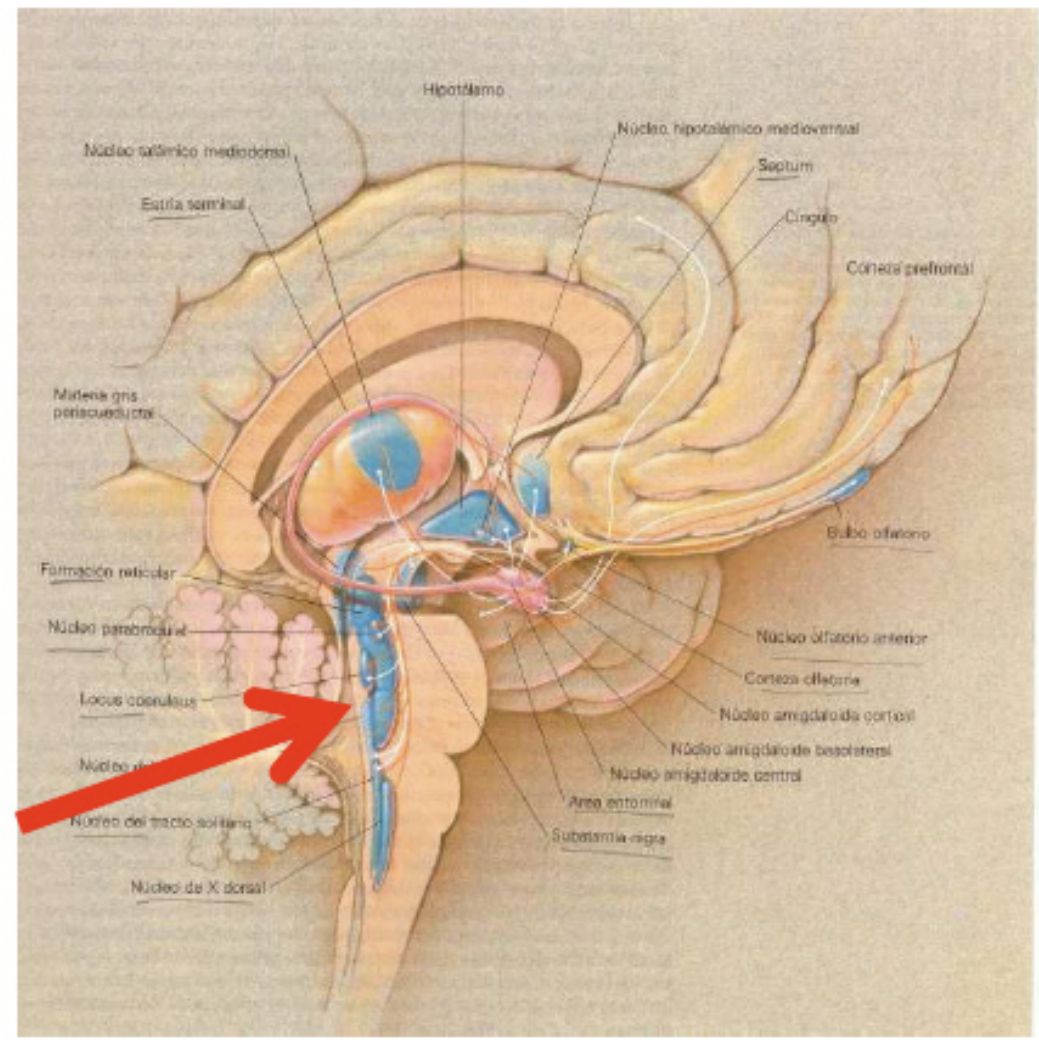

a) estructuras como el núcleo parabraquial, ligadas a la respiración, sudoración y circulación sanguínea (sistema autónomo) para preparar el organismo ante lo que se avecina (el ataque);

b) estructuras como la substantia gris periacueductal, ligadas a la movilidad (o inmovilidad);

c) estructuras como el locus coeruleus y los núcleos del Rafe, ligadas respectivamente a la producción de neurotransmisores excitatorios (como la noradrenalina), o inhibitorios (como la serotonina). La noradrenalina producirá el arousal necesario para poner el organismo en situación de atención máxima y respuesta ante el estímulo.

3. De la amígdala, tal input en bruto se dirigirá asimismo hacia estructuras del cerebro medio, como el hipotálamo, director inicial del proceso de producción de hormonas, como el cortisol. Esta hormona (conocida 
como la «hormona del estrés») pone al organismo en la situación de tensión necesaria para hacer frente al estímulo.

Hasta aquí puede hablarse de respuesta inconsciente. El organismo reacciona, actúa instintivamente ante el estímulo en bruto. «Reacción», repito, no «acción», es el término correcto para describir lo ocurrido.

Pero el proceso no termina en este punto. Precisamente aquí sucede algo importantísimo. Podría decirse que, hasta este momento, es la pura biología de la mujer en cuestión la que ha estado operando. Ahora, la cultura (o el ambiente, como se prefiera) entra en escena. Y conste que no hemos abandonado el marco en el que todo está pasando: el cerebro. Pues, para buscar la cultura no hay que salir del cerebro al mundo de ahí afuera. La cultura está inscrita y escrita en nuestro cerebro en forma de circuitos neuronales y, en particular, en forma de recuerdos. Qué sean éstos y cómo se almacenan en nuestro cerebro son cuestiones no completamente respondidas en nuestro tiempo. Es muy probable que los recuerdos sean (o se almacenen) como patrones específicos y sincronizados de actividad electroquímica en aquellas neuronas en las que se ha producido un cambio bioquímico como respuesta ante un estímulo dado. Tales neuronas se hallan en la corteza cerebral y en el hipocampo. Aunque el papel de esta última estructura en la memoria sigue debatiéndose actualmente, también es cierto que hay un amplio consenso en que son los recuerdos almacenados en él los que constituyen el entramado en el que se encajan inputs visuales como el del ejemplo que venimos analizando, un entramado, por cierto, que dota de significado emocional e histórico a tal input. Dicho de forma algo más detallada:

1. El input (la imagen en bruto, sea lo que sea ésta) que ha seguido la vía larga, adquiere forma en la corteza occipital. Pasa de ser algo amorfo a ser la imagen de algo más o menos definido. El input visual, así procesado, se dirige hacia la amígdala. En definitiva, ahora es la imagen de un hombre airado.

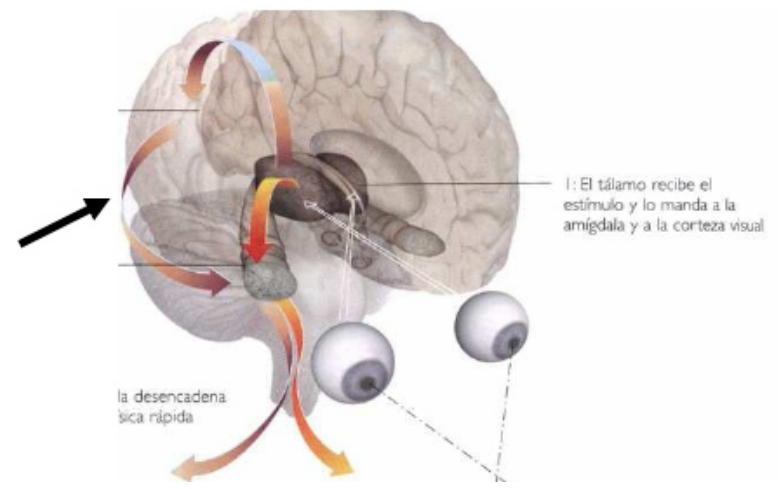


2. El hipocampo es una estructura profunda del cerebro, ubicada tras la amígdala. Como he dicho, su función, en relación con la memoria, parece ser crucial. El hipocampo le dará al input visual 'imagen de un hombre airado' un contexto histórico: lo encajará en recuerdos.

Uno de esos recuerdos podría ser el tremendo daño que ese hombre le causó a la víctima la última vez y la anterior y la anterior,... en que intentó plantarle cara. Quizá otro sea el sentimiento de inmensa soledad en que la víctima se encontró en tales circunstancias, sin familiares, sin amigos, etc., a los que acudir en busca de refugio o consuelo. Quizá otro sea el hecho de que su situación, mala, todavía empeoró tras tales intentos de hacer frente al maltrato.

Con seguridad, el hipocampo, con su recreación social o histórica del input, incidirá en la conducta de la potencial víctima: adaptarse al mal antes que afrontarlo con consecuencias aún peores. Mejor no emprender ninguna acción en ese sentido.

3. Acabo de hablar de acción y no de reacción, y no me he equivocado. El input visualmente definido y encajado en la historia (recuerdos) del compañero es objeto a su vez de una iluminación en el cerebro de la mujer: la que proviene de la corteza prefrontal. En ésta se distinguen, al menos, dos zonas cruciales para el procesamiento de un input como el descrito. Una es la zona dorsolateral, en la que parece radicar nuestra capacidad de comparar y decidir. ¿Qué hago? ¿Le pego un empujón y me marcho? ¿Le doy su merecido enfrentándome a él? ¿Le doy con la plancha que tengo en la mano?, etc. Por qué la víctima se decide a no hacer nada, a adaptarse a la conducta violenta del compañero, es seguro que tiene que tener que ver con las funciones de circuitos neuronales que han ido configurándose a base de repetir acciones parecidas. ${ }^{6}$ Ya sabemos que el cerebro es, hasta cierto punto, plástico.

En definitiva, el input, visualmente definido por la acción de la corteza occipital, y encajado en la historia del individuo en cuestión por la intervención del hipocampo, recibe en la amígdala la iluminación, en suma, de sus ideas y creencias en un proceso de comparación de alternativas y de toma de decisiones, que no es otra cosa a su vez que el resultado de la función de determinados circuitos neuronales en la corteza prefrontal. La conducta de la potencial víctima es el resultado de un conjunto de interacciones entre la biología y la cultura sin abandonar el escenario del cerebro.

6 A la hora de ejecutar la decisión tomada acerca de qué conducta poner en práctica (enfrentarse o adaptarse) entrará en juego otra área de la corteza prefrontal: la orbitofrontal. 
Para reflejar este nivel de explicación neurobiológica trazaré un rectángulo en la parte inferior de la palabra «sumisión», así:
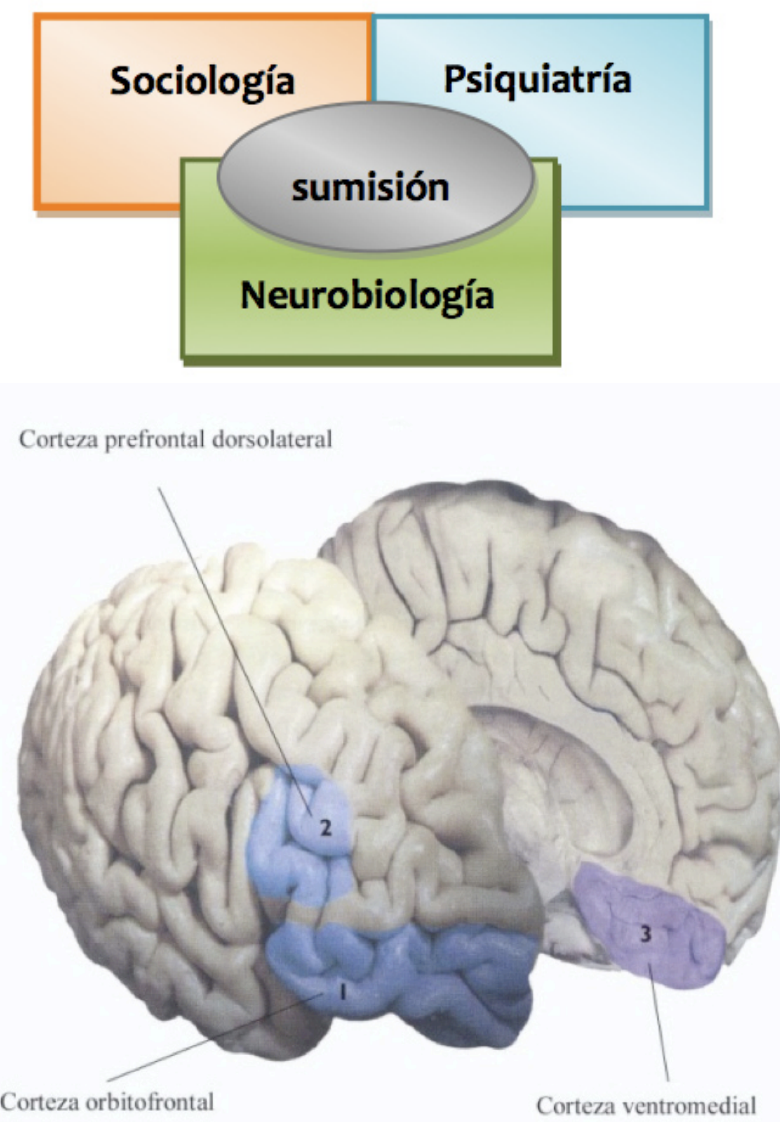

Ignorar este nivel de complejidad más profundo quizá es lo que lleva a ciertos psiquiatras y psicólogos a responsabilizar a las víctimas de lo que les sucede y a exigirles una conducta que, en la realidad, exige la reconfiguración de parte de su biología cerebral. Lo peor es que pueden creer que el fármaco basta, exactamente igual que los sociólogos pueden creer que es suficiente con cambios sociales. No son suficientes, aunque sí necesarios.

Lo que hay que cambiar además son los recuerdos incluyendo entre ellos, claro está, los relacionados con el poco o nulo éxito de las conductas puestas en práctica para salir de la situación de maltrato. Así pues, esa reconfiguración entraña una intervención social, sí, pero acompañada de la psicoterapia pertinente. 
Y todo esto se pierde de vista cuando uno se acerca desde la perspectiva unilateral de una ciencia determinada a un proceso tan complejo como el descrito. Dicho de otro modo, la división del saber en territorios disjuntos es interesadamente útil para que los especialistas marquen distancias y determinen el ámbito en el que anhelan tener mando en plaza, pero es contraria a lo que, en el plano de la realidad, ${ }^{7}$ sucede.

\section{VI. «PERO, ¿POR QUÉ LE HABRÉ DICHO QUE Sí? Si YA HABÍA DECIDIDO ODIARLO POR} TODA LA ETERNIDAD»

Después de lo dicho, me atrevería a aseverar que ya está bien de prejuicios por ambas partes. Los ambientalistas siguen firmes en sus creencias de que la biología (me refiero en concreto a la biología humana) es plastilina, moldeable prácticamente sin límite. Siguen firmes asimismo en sus descalificaciones de cuantos aluden a la biología como un condicionante. Dada la ligazón entre ambientalismo y ciertas corrientes político-ideológicas, quienes hablan de biología suelen ser tildados de «reaccionarios». No deja de ser estúpido.

A mí me preocupa científicamente la sumisión de algunas personas ante sus agresores. Me gustaría encontrar una explicación para el hecho, descrito antes, de que algunas mujeres, que sufren violencia de género, ni se enfrentan a sus agresores, ni huyen. ¿Basta acudir a factores sociales para explicar una

7 Por dos veces en poco tiempo, he aludido a la realidad. Lo he hecho a sabiendas. No diré, tan rotundamente como Bunge, que somos tolerantes con los antirrealistas y analizamos sus sofismas, mientras que a quienes en la vida cotidiana pierden contacto con la realidad los sujetamos a tratamiento psiquiátrico. Exactamente, dice Bunge (1985, págs.. 41-42): «en la vida cotidiana compadecemos o tememos a quienes pierden contacto con la realidad, y despreciamos a quienes la falsean deliberadamente. Tildamos de mentirosos a éstos últimos, y deseamos que los primeros sean sometidos a tratamiento psiquiátrico. En cambio, en la vida académica hacemos gala de tolerancia para con los antirrealistas. Analizamos pacientemente los sofismas de los filósofos que sostienen que el mundo es un sueño o un conjunto de sensaciones, o que las teorías científicas son ficciones, o metáforas, o convenciones comparables con los sistemas de señales. Tomamos en serio a quienes pretenden que no hay diferencia entre ciencia y seudociencia, entre hipótesis comprobada y especulación desenfrenada, entre dato experimental y mito.» Creo que los argumentos de los antirrealistas parten a menudo de una base sólida. Tal vez lo que les sucede a muchos de ellos es que se pierden luego en un laberinto de sutilezas. En concreto esa base sólida puede proporcionarla la idea de que, desde un punto de vista cognitivo, no somos pasivos. Somos activos. Conocemos lo que nuestras categorías, esquemas conceptuales, etc., nos permiten conocer y lo conocemos como estos elementos nos lo permiten conocer. Estoy plenamente de acuerdo: conocemos lo que nos permiten conocer tales entramados, pero debe haber un «lo que», una realidad que, ciertamente, nosotros remodelamos de acuerdo con las características de nuestro aparato cognitivo. Unas características, por cierto, que en buena medida serán el resultado de un proceso de mutación y selección en el que la realidad jugará un papel crucial. Aquí entran en juego nuevamente las interacciones entre ambiente y biología. 
conducta de este tipo? ¿Qué tiene de malo que, aceptando el papel que estos factores sociales (por ejemplo, la adopción de estereotipos y prejuicios sexistas por parte de la víctima) pueden desempeñar, qué tiene de malo (repito) recurrir al análisis psiquiátrico-psicológico para identificar posibles síndromes, como el de indefensión aprendida? Y, ¿qué tiene de malo analizar los procesos neurofisiológicos por los que se fijan en nuestra memoria tales estereotipos y prejuicios, junto a creencias distorsionadas acerca de las iniciativas que se pueden acometer?

Y conste que no estoy diciendo que unos factores se reduzcan totalmente a otros. No estoy afirmando que elementos de orden superior pueden ser perfectamente descartados y substituidos por elementos de orden inferior, sin pérdida de capacidad explicativa. No estoy afirmando, en definitiva, que algún día la sociología se reducirá a psicología, la psicología a biología y ésta a su vez a la físico-química. Estoy muy lejos de entender la consiliencia bajo la perspectiva de un micro-reduccionismo de ese tipo, de un reduccionismo depredador. Sólo estoy aseverando algo de sentido común: que en el plano de la realidad hay interacciones ontológicas que, en el plano científico, deberían ser atendidas por interacciones epistémicas entre diversas teorías. Ése es el tipo de consiliencia deseable. A la vez digo rotundamente, eso sí, que proceder de este modo, dilucidar esas interacciones ontológicas en el marco de interacciones teórico-científicas, no sólo es lo coherente desde un punto de vista metodológico: es también lo más fructífero si se tienen en cuenta los grandes desarrollos que han tenido ciencias cuyos nombres ya delatan su carácter consiliente. Me he referido antes a la bio-química, la psicobiología y similares. Ahora añado que deberíamos prestar especial atención en este contexto a la neurociencia cognitiva como ámbito del saber que fomenta la colaboración de investigadores de áreas tan dispares como la neurofisiología, la ecología del comportamiento, la informática, la genética, la psicología y la psiquiatría.

\section{REFERENCIAS BIBLIOGRÁFICAS}

BREDO, E. 2000: «Unifying Biological and Cultural Psychology», The Journal of the Learning Sciences 9(2): 221-232.

BUNGE, M. 1985: Racionalidad y realismo. Madrid: Alianza Universidad.

CRAVER, C.F. 2007: Explaining the Brain: Mechanisms and the Mosaic Unity of Neuroscience. Oxford: Clarendon Press.

DARDEN, L. \& CRAVER, C.F. 2009: «Reductionism in Biology», en Encyclopedia of the Life Sciences. Chichester: John Wiley \& Sons, Ltd. 
DENNET, D. 1995: Darwin's Dangerous Idea: Evolution and the Meanings of Life. New York: Simon \& Schuster.

GLIMCHER, P. W. \& RUSTICHINI, A. 2004: «Neuroeconomics: the Consilience of Brain and Decision», Science 306(5695): 447-452.

JACKSON, T. 2002: «Evolutionary Psychology in Ecological Economics: Consilience, Consumption and Contentment», Ecological Economics 41: 289-303.

PICCININI, G. \& CRAVER, C. 2011: «Integrating Psychology and Neuroscience: Functional Analyses as Mechanism Sketches», Synthese 183:283-311.

RAND, K. L. \& ILARDI, S. 2005: «Toward a Consilient Science of Psychology», Journal of Clinical Psychology 61(1): 7-20.

SANMARTÍN ESPLUGUES, J. 2000: La violencia y sus claves. Barcelona, Ariel (6 ed., 2009) (Nuevamente publicado en la colección Quintaesencia de Ariel, $7^{\mathrm{a}}$ ed. actualizada, 2013) , 2002: La mente de los violentos. Barcelona: Ariel. , (ed.) 2004: El laberinto de la violencia. Barcelona: Ariel. , (en prensa): «Claves de la violencia en el siglo XXI», Ludus vitalis.

SHELDON, K. M. 2011: «Consilience within the Biopsychosocial System», Psychological Inquiry: An International Journal for the Advancement of Psychological Theory 22: 52-65.

SLINGERLAND, E. \& COLLARD, M. (eds.) 2011: Creating Consilience. Integrating the Sciences and the Humanities. Oxford: Oxford University Press.

WILSON, E. O. 1998: Consilience: The Unity of Knowledge. New York: Alfred A. Knopf (version digitalizada: https://files.nyu.edu/mr185/public/www/classes/ readings/Wilson\%20-\%20Consilience\%20The\%20Unity\%20of\%20Knwoledge1. pdf)

[Versión castellana: Consiliencia: la unidad del conocimiento. Barcelona: Círculo de Lectores; Galaxia Gutenberg, 1999]

José SANMARTín Esplugues es catedrático de Lógica y Filosofía de la Ciencia (en excedencia) de la Universitat de València y director de la Oficina para el Desarrollo del Campus Virtual de la UCV-San Vicente Mártir.

Publicaciones recientes:

2009: La violencia y sus claves (Ariel 2000, $6^{\mathrm{a}}$ edición).

2008: El enemigo en casa (Nabla).

Correo electrónico: jsanmartinesplugues@gmail.com.

Algunas direcciones de interés:

http://es.wikipedia.org/wiki/Jos\%C3\%A9_Sanmart\%C3\%ADn_Esplugues, http://www. issuu.com/jsanmartin 